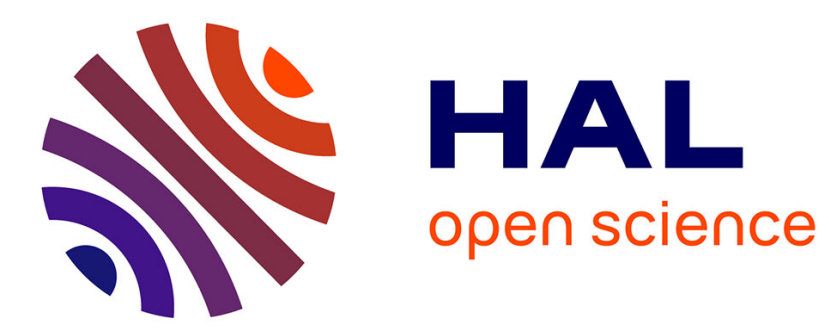

\title{
Un assemblage au nom d'Amenemhat Ier dans les magasins du temple de Louxor
}

\author{
Luc Gabolde
}

\section{To cite this version:}

Luc Gabolde. Un assemblage au nom d'Amenemhat Ier dans les magasins du temple de Louxor. Peter Brand et Louise Cooper. " Causing his name to live ", Studies in Egyptian Epigraphy and History in Memory of William Murnane, 37, Brill, pp.103-107, 2009, Culture and History of the Ancient Near East, 978-90-47-42988-3. halshs-00751767

\section{HAL Id: halshs-00751767 https://shs.hal.science/halshs-00751767}

Submitted on 7 Jun 2018

HAL is a multi-disciplinary open access archive for the deposit and dissemination of scientific research documents, whether they are published or not. The documents may come from teaching and research institutions in France or abroad, or from public or private research centers.
L'archive ouverte pluridisciplinaire HAL, est destinée au dépôt et à la diffusion de documents scientifiques de niveau recherche, publiés ou non, émanant des établissements d'enseignement et de recherche français ou étrangers, des laboratoires publics ou privés. 


\title{
Un assemblage au nom d'Amenemhat $\mathrm{I}^{\mathrm{er}}$ dans les magasins du temple de Louxor
}

\author{
Luc Gabolde, \\ Centre Franco-Égyptien pour l'étude des Temples de Karnak
}

En 1999, Bill Murnane, en réponse à une question que je lui posais sur l'ancienneté du temple de Louxor, m’avait révélé l'existence des documents jusqu'alors inédits que j’ai le privilège de présenter ici en hommage à sa science et à son humanité.

Il s’agit d'un lot de blocs en calcaire décorés en bas-reliefs et portant des éléments de la titulature d'Amenemhat $\mathrm{I}^{\mathrm{er}}$ (fig. 1). Conservés dans le magasin sud du temple de Louxor, ils ont été minutieusement documentés en 1996 par le Dr. R. Johnson et l'équipe de l'Oriental Institute ${ }^{1}$ et portent aujourd'hui les n d'inventaire ES 393 (situé à l'est de $123 \mathrm{E}$ ) et nº 875 (situé en 122 W, extrémité sud). L'assemblage est composé de deux très longs blocs — chacun brisé en deux parties - qui se raccordent quasi directement pour former les restes d'une scène. Ce format est étrange et résulte apparemment du débitage du ou des blocs primitifs en longs moellons, débitage qui doit avoir été effectué à la scie après le démantèlement de l'édifice auquel ils avaient appartenu. ${ }^{2}$ Le bloc supérieur comporte en outre la trace d'une sorte d'échancrure, réalisée à coups de ciseaux et de scie, comme si une pièce d'encastrement y avait été primitivement insérée. Quoi qu'il en soit, le bloc d'origine était assez peu épais et devait former une sorte de placage de pierre plutôt qu'une maçonnerie pleine. Il faudrait donc imaginer un édifice originel constitué d'un noyau de maçonnerie en grès ou en briques crues et recouvert d'un placage de calcaire portant la décoration. ${ }^{3}$

\section{Le matériau}

Les blocs sont taillés dans une pierre calcaire. La nature exacte du matériau utilisé n’est pas facile à déterminer sur les seules indications données par un examen de la photo et il ne m’a pas été possible de procéder à un examen de visu de la roche. Sa cassure paraît grumeleuse et l'apparente en cela au calcaire de Tourah extensivement employé plus tard par Sésostris $\mathrm{I}^{\mathrm{er}}{ }^{4}{ }^{4}$ Les

${ }^{1}$ Avec une libéralité dont je lui suis profondément reconnaissant, le Dr. Raymond Johnson, directeur du Chicago House de Louxor, a accepté de m'en confier les photos assorties de l'autorisation (de ses encouragements, devrais-je dire) de les publier ici. Son recensement des blocs du Moyen Empire conservés dans les magasins de Louxor comporte 22 autres blocs, parmi lesquels on relève un élément avec un cartouche de Sésostris III et deux autres avec le simple nom de Sésostris.

${ }^{2}$ Le débitage en long blocs, sans doute pour obtenir des éléments de linteau, de jambages ou de seuil, est encore attesté pour des blocs du Moyen Empire conservés dans le magasin du Cheikh Labib à Karnak : 87 CL 57 ; 87 CL 59 ; 87 CL 61 ; 87 CL 300. Un élément similaire — sur lequel on reviendra plus bas — a été retrouvé à Tôd : L. Postel, « Fragments inédits du Moyen Empire à Tôd (mission épigraphique de l'IFAO) », dans J.-Cl. Goyon, Chr. Cardin (éd.), Actes du IX Congrès international des égyptologues, Grenoble, 6-13 septembre 2004, OLA 150, (Louvain : Peeters, 2006), p. 1539-1550.

${ }^{3}$ À Éléphantine, plusieurs monuments de la $\mathrm{XI}^{\mathrm{e}}$ dynastie avaient ainsi été constitués de briques crues plaquées de parois en grès, voir W. Kaiser et alii, " Stadt und Tempel von Elephantine, 19./20. Grabungsbericht », MDAIK 49, 1993, p. 148-152 ; idem. « Stadt und Tempel von Elephantine, 25./26. Grabungsbericht », MDAIK 55 (1999), p. 90-94.

${ }^{4}$ Sur le calcaire employé par de Sésostris I ${ }^{\mathrm{er}}$, à Karnak, voir Ch. Karlshausen et Th. De Putter, « Provenance et caractères distinctifs des calcaires utilisés dans l'architecture du Moyen et du Nouvel Empire à Karnak », Karnak 11 (Paris : ERC, 2003), p. 373-386. 
constructions d'Amenemhat $\mathrm{I}^{\mathrm{er}}$ à Ermant avaient toutefois été réalisées dans un calcaire local ${ }^{5}$ tout comme celles qui lui sont attribuables à Karnak. ${ }^{6}$

\section{Le style}

Le bloc est traité en bas-relief très peu saillant, difficilement comparable, de ce point de vue, aux décors en relief dans le creux du même Amenemhat $\mathrm{I}^{\mathrm{er}}$ à Ermant ${ }^{7}$ et notablement distinct des décors en méplat assez saillant de Sésostris $\mathrm{I}^{\mathrm{er}}$ à Karnak. Ce style en relief très peu marqué se rattache à la tradition de l'Ancien Empire, mais aussi aux styles en cours à la fin de la $\mathrm{XI}^{\mathrm{e}}$ dynastie comme celui des reliefs raffinés de Mentouhotep III-Seânkhkarê à Tôd, Ermant et Éléphantine. ${ }^{8}$ On relève que ce sera encore la tradition de l'atelier d'artisans qui a réalisé les bas-reliefs de Sésostris I ${ }^{\text {er }}$ à Éléphantine. ${ }^{9}$

Le style et l'épigraphie sont sobres et dépourvus des abondants détails que l'on rencontre sur les décors d'Amenemhat $\mathrm{I}^{\mathrm{er}}$ à Ermant, sur ceux de Mentouhotep III à Tôd ou, plus tard, sur ceux de Sésostris $\mathrm{I}^{\mathrm{er}}$ à Karnak.

\section{La scène}

Les éléments fragmentaires dont on dispose permettent de restituer une scène où le roi, coiffé de la couronne rouge, se dirigeait vers la gauche, précédé d'une enseigne d'Oupouaout ${ }^{10}$, comme c’est souvent le cas lors des sorties solennelles, lors des «montées royales » ${ }^{11}$ ou encore pendant les cérémonies jubilaires. ${ }^{12}$ Il serait hautement spéculatif d'essayer ici de la reconstituer plus complètement.

${ }^{5}$ Pour les blocs d’Amenemhat I ${ }^{\mathrm{er}}$ trouvés à Ermant voir R. Mond, O.H. Myers, Temples of Armant I-II, EEM 43 (Londres : Egypt Exploration Society, 1940), pl. LXXXVIII et les dessins pl. XCVIII. C’est mon propre examen visuel des pierres d'Ermant qui m'a conduit à la conclusion qu'il s'agissait d'une roche locale. Des analyses plus poussées seraient les bienvenues pour confirmer ou infirmer cette estimation.

${ }^{6}$ Un bloc en calcaire avec une représentation d'Atoum, remployé dans la plate-forme en grès de la « cour du Moyen Empire », doit appartenir au décor du temple d'Amenemhat $\mathrm{I}^{\mathrm{er}}$. Il s'agit apparemment d'un calcaire local (voir L. Gabolde, J.-Fr. Carlotti, E. Czerny, « Aux origines de Karnak : les recherches récentes du Centre FrancoÉgyptien d’Étude des Temples de Karnak dans la “ cour du Moyen Empire ” », BSEG 23 (1999), p. 40, fig. 8-9-11). Les pierres utilisées à Karnak au Moyen Empire ont été, on l’a mentionné, étudiées par Ch. Karlshausen et Th. De Putter ( Provenance et caractères distinctifs des calcaires utilisés dans l'architecture du Moyen et du Nouvel Empire à Karnak », Karnak 11 (Paris : ERC, 2003), pp. 373-385). L’échantillonnage examiné ne comportait pas d’élément d'Amenemhat $\mathrm{I}^{\mathrm{er}}$ mais du règne suivant, celui de Sésostris $\mathrm{I}^{\mathrm{er}}$, lequel emploie exclusivement le calcaire de Tourah.

${ }^{7}$ Supra n. 5.

${ }^{8}$ F. Bisson de la Roque, Tôd 1934 à 1936, FIFAO XVII (Le Caire : Imprimerie de l’Institut Français d'Archéologie Orientale, 1937), pls. XXI,2-XXVII et R. Mond, O. H. Myers, Temples of Armant, pl. LXXXVIII (Éléphantine) et XCIV-XCVII (Ermant).

${ }^{9}$ L. Habachi, « Building Activity of Sesostris I in the Area South of Thebes », MDAIK 31 (1975), pl. 12 ab. Voir encore W. Kaiser et alii, « Stadt und Tempel von Elephantine, 13/14. Grabungsbericht », MDAIK 43 (1986), p. 84-88 et pl. 8 .

${ }^{10}$ Sur cette enseigne, qui représente le nom d’Horus du roi, voir P. Barguet, « Un groupe d’enseignes en rapport avec les noms du roi », RdE 8 (1951), p. 9-19.

${ }^{11}$ Voir, sous Sésostris I ${ }^{\mathrm{er}}$, l'introduction du roi dans la chapelle d'Anubis, ou un des rituels préludant à l'érection du mât de Min, P. Lacau, H. Chevrier, Une chapelle de Sésostris $I^{\text {er }}$ à Karnak (Le Caire : Imprimerie de l'Institut Français d'Archéologie Orientale, 1956-1969), pl. 13, scène 3 ; pl. 31, scène 8', ou bien, pour des occurrences plus communes et plus récentes, H.H. Nelson, W. Murnane, The Great Hypostyle Hall at Karnak, vol. I, part 1, The Wall Reliefs, OIP 106 (Chicago : Oriental Institute of the University of Chicago, 1981), pl. 50 ; 51.

${ }^{12}$ F.W. F. von Bissing, Das Re-Heiligtum des Königs Ne-woser-re (Rathures) II (Leipzig : Hinrichs, 1923), pl. $1,10,11$ et particulièrement 13 . 
Les textes

Devant le roi, sa légende :

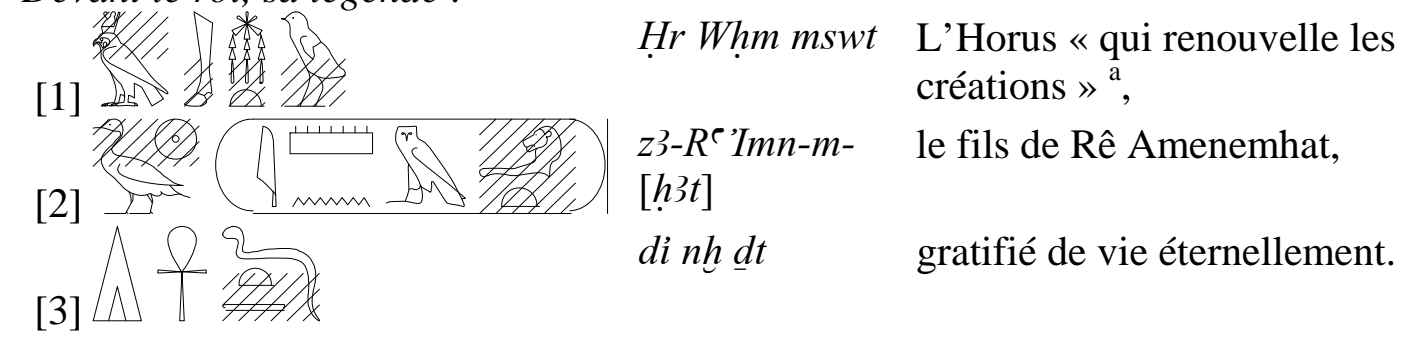

Au-dessus de la déesse vautour (dont on aperçoit l'extrémité des plumes) :

[4]
Nhbt
Nekhbet ${ }^{\mathrm{b}}$.

a - Il s’agit du second nom d’Horus du roi, adopté apparemment après quelques années de règne. ${ }^{13}$ Il faut peut-être comprendre que ce nom-programme signale la mise en œuvre d'un vaste projet de construction du roi, le mot $m s w t$ ( « ce qui a été créé = création ») pouvant, en effet, signifier sporadiquement « création(s) architecturale(s) ». ${ }^{14}$ La titulature viendrait ainsi faire écho à la trace architecturale laissée par les blocs, lesquels constituent par eux mêmes des indices de cette refondation proclamée.

b - On remarquera que le nom et l'image de la déesse n’ont pas été attaqués par les agents d'Akhenaton, contrairement à ce que l'on constate globalement sur le territoire thébain, ce qui montre que ces bloc étaient hors d'atteinte au Nouvel Empire (parce que sans doute remployés).

\section{La provenance des blocs et la question de l'ancienneté de Louxor}

La présence de ces vestiges au nom d'Amenemhat $\mathrm{I}^{\mathrm{er}}$ conduit naturellement à s'interroger sur leur provenance exacte et, subséquemment, sur l'ancienneté des sanctuaires de Louxor. Ces blocs mis à part, les plus anciens témoins historiques exhumés du site du temple sont constitués d'une table d'offrandes de Sésostris III, usurpée par Apopi, ${ }^{15}$ d'une seconde table d'offrandes, au quadruple nom d'un Sésostris indéfini, trouvée dans le village ${ }^{16}$, enfin d'éléments de porte de Sobekhotep II. ${ }^{17}$ Comme le soulignait déjà G. Daressy, la table d’offrandes de Sésostris III pourrait bien avoir été apportée d’Hérakléopolis en raison des divinités qui y sont mentionnées (et ce, bien après le Moyen Empire). Les éléments trouvés dans le village peuvent, quant à eux,

${ }^{13}$ J. von Beckerath, « Zur Begründung der 12. Dynastie durch Ammenemes I. », ZÄS 92 (1966), p. 7 ; idem., Handbuch der ägyptischen Königsnamen, MÄS 49 (Mayence : Philip von Zabern, 1999), p. 83 et p. 82 , n. 1.

${ }^{14}$ Chr. Wallet-Lebrun, « Contribution à l’histoire de la construction à Karnak », dans L'égyptologie, histoire, résultats et perspectives (Grenoble : Presses Universitaires de Grenoble, 1994), p. 227-229 ; voir les mentions éloquentes dans les récits de refondation de temples de Thoutmosis III : Urk. IV, p. 817, 10 et 17 ; 820, 17 ; 830, 8 .

${ }^{15}$ PM II $^{2}$, p. 339 = CG 23009, A.B. Kamal, CGC, Tables d'offrandes (Le Caire, 1906-09), pl. V, p. 8-9 et E. Grébaut, « Fouilles de Louqsor », BIE X (1889), p. 335-6.

${ }^{16} \mathrm{PM} \mathrm{II}^{2}$, p. 339 = K. Lepsius, Denkmäler aus Ägypten und Äthiopien, Text III (Genève : Editions des Belles-Lettres, 1975), p. 89.

${ }^{17} P M \mathrm{II}^{2}$, p. 338 = G. Daressy, « Le voyage d'inspection de M. Grébaut en 1889 », ASAE 26 (1926), p. 8. 
provenir aussi bien des ruines de Karnak que de celles de Louxor, sans compter qu'on a pu encore les transférer de la rive gauche.

Les deux fragments architecturaux en granit rose de (Sekhemrê-khoutaouy)-Sobekhotep II qui furent exhumés dans la cour d'Amenhotep III sont peut-être plus significatifs, mais ils sont bien incapables de garantir une ancienneté du site allant au-delà de leur époque.

S’il est vraisemblable, malgré tout, que la fondation des sanctuaires de Louxor remonte au moins au Moyen Empire, compte tenu du conservatisme théologique des anciens égyptiens, force est, cependant, de constater qu'aucun vestige n’en a jamais été trouvé in situ, et donc que l’hypothèse demeure une pure, quoique séduisante, conjecture.

\section{Un matériau de récupération amené d'ailleurs?}

Compte tenu du débitage qu'ont subi les blocs, on est tenté d’y reconnaître un matériau de récupération, amené sur le site après l'abandon des cultes. ${ }^{18}$ Ce genre de transfert est bien attesté pour la plupart des sites de la région thébaine : des talatates de Karnak se sont ainsi retrouvées au temple de Tôd, ${ }^{19}$ d'autres ont rejoint Ermant. ${ }^{20}$ À Louxor, c'est encore de Karnak que provient l'essentiel des blocs arrachés d'un autre site. On recense ainsi dans les magasins de Louxor la série d'éléments suivants originaires du grand temple d'Amon-Rê :

$1^{\circ}$ ) un fragment d'obélisque en granit rose de Thoutmosis $\mathrm{I}^{\mathrm{er}}$ provenant de l'obélisque nord du IV $\mathrm{e}^{\mathrm{e}}$ pylône de ce roi (inédit);

$2^{\circ}$ ) plusieurs fragments des obélisques orientaux d'Hatchepsout (avec notamment les figures des Amon ithyphalliques), généralement remployés en meules (inédits) ;

$3^{\circ}$ ) des talatates d'Akhenaton dont les thèmes permettent de les raccorder aux talatates remployées dans les murs des cours des $\mathrm{IX}^{\mathrm{e}}-\mathrm{X}^{\mathrm{e}}$ pylônes (et d’une manière générale toutes les talatates retrouvées à Louxor) ;

$4^{\circ}$ ) une table d'offrandes de Thoutmosis III provenant manifestement de l'Akhmenou. ${ }^{21}$

\section{L'éventualité d'une origine à Karnak}

Or, à Karnak, les vestiges au nom d'Amenemhat $\mathrm{I}^{\mathrm{er}}$ sont, justement, bien attestés, montrant que l'activité architecturale de ce roi y avait été loin d'être négligeable. Il y a tout d'abord le très important socle de naos en granit rose destiné à recevoir le tabernacle de la

${ }^{18}$ Dans le magasin dit du « Cheikh Labib » à Karnak sont conservés des blocs du Moyen Empire en calcaire dont le format actuel, très allongé, peu haut et peu épais, semblable à celui des blocs d'Amenemhat $\mathrm{I}^{\mathrm{er}}$ traités ici, semble indiquer qu'ils avaient été débités pareillement, dans la perspective d'une réutilisation. Voir leur liste, supra n. 2.

${ }^{19}$ M. Etienne dans G. Pierrat et alii, « Fouilles du Musée du Louvre à Tôd, 1988-1991 », Karnak X (Paris : ERC, 1995), p. 490-492. Les blocs proviennent assurément de Karnak et ont probablement été amenées à Tôd à l'époque ptolémaïque ou romaine.

${ }^{20}$ Comme l’a rappelé A. Eggebrecht (« Armant », LÄ I, col. 437), l’éventualité que les quelques fragments du règne d’Amenhotep IV trouvés à Ermant (R. Mond, O. H. Myers, The Temple of Ermant ; Idem, Bucheum II, EEM 41 (Londres : Egypt Exploration Society, 1934), p. 46) puissent effectivement attester l'existence d'un temple primitif d'Aton dans cette ville et que celle-ci ait eu un rôle particulier dans l'essor de la nouvelle théologie a été mise en doute avec des arguments de fond par H. Kees (« Ein Sonnenheiligtum im Amonstempel von Karnak », Orientalia 18 (1949), p. 433 et 439).

${ }^{21} P M \mathrm{II}^{2}$, p. $339=\mathrm{L}$. Habachi, «Clearance of the Area to the East of Luxor Temple and Discovery of Some Objects », ASAE 51 (1951), pl. V, fig. 12 et p. 464-68 [IX]. 
vénérable statue d'Amon. ${ }^{22}$ Aujourd'hui remployé au temple de Ptah, il ne fait guère de doute qu'il avait à l'origine été installé dans le sanctuaire qui s’était dressé dans la « cour du Moyen Empire ». À ce très ancien temple d'Amon appartient sans doute le fragment de bloc décoré en relief dans le creux qui avait été remployé dans la plate-forme en grès. Représentant une scène d'allaitement du roi en présence du dieu Atoum, il pourrait, en effet, remonter au règne d'Amenemhat I ${ }^{\mathrm{er}} .{ }^{23}$ Le second élément d'importance est un groupe statuaire fragmentaire, une dyade, en granit gris ayant originellement figuré Amon et le roi. ${ }^{24}$ On peut encore suspecter que le groupe statuaire à six personnages trouvé près de l'autel solaire au nord de la « cour du Moyen Empire » lui soit redevable. ${ }^{25}$ Ces documents font d'Amenemhat $\mathrm{I}^{\mathrm{er}}$ un des souverains les plus actifs sur le site de Karnak avant le règne de Sésostris $\mathrm{I}^{\mathrm{er}}$; il n’y aurait rien d'extraordinaire à ce que des parois de placage en calcaire décorées en bas relief aient orné les murs du temple primitif où il avait installé son naos. ${ }^{26}$ Démontés par Sésostris $\mathrm{I}^{\mathrm{er}}$, ces éléments auraient ensuite pu servir à garnir les fondations de son nouveau temple. Réapparus après la désaffection des cultes et le démantèlement du temple par les chaufourniers médiévaux, les placages de calcaire auraient ensuite été apportés, entre autres, à Louxor et débités en éléments longs, constituant ces éléments mêmes qui ont été retrouvés et documentés par l’équipe de l’Oriental Institute.

\section{Des blocs isolés ?}

Une ultime interrogation demeure : l'assemblage de blocs d'Amenemhat $\mathrm{I}^{\mathrm{er}}$ conservé dans les magasins de Louxor est-il seul de son espèce ? En fait, un bloc des magasins du temple de Tôd, dépourvu de nom royal, mais pareillement décoré en bas-relief, présente des dimensions, un style de décor et pour finir un type de retaille en forme de longue poutre, extrêmement similaires à ce que l'on observe sur l'assemblage qui nous occupe ${ }^{27}$; peut-être a-t-il été lui aussi arraché à Karnak, comme l’ont été bien d'autres éléments retrouvés sur ce site. ${ }^{28}$

${ }^{22} P M \mathrm{II}^{2}$, p. 200 (23) = A. Mariette, Karnak : étude topographique et archéologique (Leipzig : Heinrichs, 1875), p. 10, 41-42, pl. 8 e ; E. Hirsch « Die Kultpolitik Amenemhets I. im Thebanischen Gau », dans Ägyptische Tempel-Struktur, Funktion und Programm, HÄB 37 (Hildesheim : Gerstenberg Verlag, 1994), p. 137-142, notamment p. 139.

${ }^{23}$ Supra, n. 6.

${ }^{24}$ PM II ${ }^{2}$, p. 107 ; G. Evers, Staat aus dem Stein (Munich : Bruckmann, 1929), p. 95, § 634, p. 22, ill. 4 et pl. II, ill. 35 ; A. Mariette, Karnak, p. 41, n 4, pl. 8 d ; G. Legrain, « Notes prises à Karnak », RecTrav 23 (1901), p. 63 ; M. Seidel, Die Königlische Statuengruppen I, HÄB 42 (Hildesheim : Gerstenberg Verlag, 1996), p. 65-66 (doc. 31).

${ }^{25} \mathrm{PM} \mathrm{II}^{2}$, p. 103, (307) ; M. Seidel, Die Königlische Statuengruppen I, doc. n 32, p. $67-68$ et pl. 23 a-c et L. Gabolde, J. Fr. Carlotti et E. Czerny, Trois études sur le Moyen Empire à Karnak à paraître. Il pourrait se raccorder à un élément similaire acheté par Mond et Myers à Tôd.

${ }^{26}$ Les fouilles récentes du CFEETK dans le secteur des cours nord et sud du VII pylône ont confirmé l'existence de structures de briques crues contemporaines ou de très peu antérieures aux aménagements de Sésostris $\mathrm{I}^{\mathrm{er}}$ et ont montré aussi que la stratigraphie du lieu ne pouvait guère remonter au-delà de la fin de la PPI ou du début de la XI ${ }^{\mathrm{e}}$ dynastie (G. Charloux, J.-Fr. Jet, E. Lanoë, « Nouveaux vestiges des sanctuaires du Moyen Empire à Karnak. Les fouilles récentes des cours du VI pylône », BSFE 160 (2004), p. 26-46).

${ }^{27} \mathrm{~N}^{\circ}$ d'inventaire : Tôd 310. Ma gratitude va à L. Postel qui publie ce bloc avec les autres trouvailles du site (« Fragments inédits du Moyen Empire à Tôd (mission épigraphique de l'IFAO) », dans J.-Cl. Goyon, Chr. Cardin (éd.), Actes du IX Congrès international des égyptologues, Grenoble, 6-13 septembre 2004, OLA 150 (Louvain : Peters, 2006), p. 1539-1550) et qui m’a permis de faire état ici, en primeur, de son existence.

${ }^{28}$ Supra n. 19. 


\section{Conclusion provisoire}

Les blocs isolés au nom d'Amenemhat $\mathrm{I}^{\mathrm{er}}$ trouvés sur le site de Louxor et enregistrés dans les magasin du CSA, de par leur format et du fait qu'ils montrent des traces de débitage, ont sans doute été apportés comme matériau de construction depuis un autre site de la région, comme ont, par exemple, été apportés à Louxor les talatates qui y ont été découvertes. Compte tenu des traces conséquentes de l'activité architecturale d'Amenemhat $\mathrm{I}^{\mathrm{er}}$ à Karnak, ce dernier site constitue probablement le meilleur candidat pour leur provenance et ils en constitueraient quelques uns des plus anciens vestiges.

\section{Résumé en Anglais}

Two broken limestone slabs, stored in the Luxor temple magazine and carefully documented by the team of the Chicago Oriental Institute, bear fragments of the titulatury of king Amenemhet I. As the blocks have apparently been sawn and recut, they can be considered as reused material, and may have been brought from other sites in the Theban area. It is a matter of fact that several pieces found in the vicinity of Luxor Temple precisely originate from Karnak. It is also well known that Amenemhet I worked at Karnak and, significantly, dedicated a granite naos there for the sacred sanctuary of the god Amun-Râ. On the other hand, no archeological element can guarantee that the temple of Luxor could be older than the reign of Sobekhotep II, and nothing there can, of course, be related to Amenemhat I. I would consider it plausible that the two blocks published here in memory of Bill Murnane - who had mentioned their existence to me- constitute the remains of one of the oldest temples dedicated to Amun at Karnak. They would have reappeared after the dismantling of the sanctuary and the subsequent recovery of the building material, which was then brought to the site of Luxor.

\section{Figures}

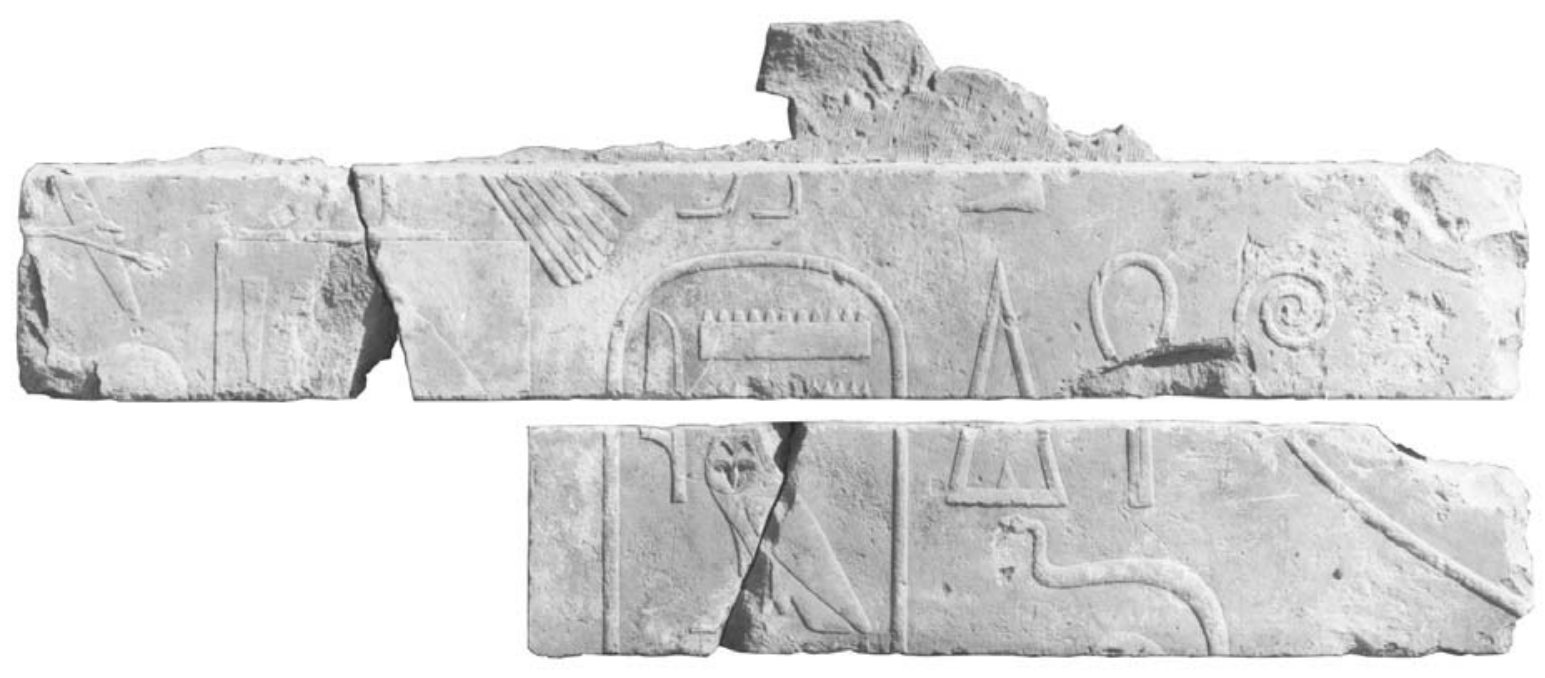

0 100

Fig. 1 : Assemblage de blocs d'Amenemhat $\mathrm{I}^{\mathrm{er}}$ dans les magasins du temple de Louxor 